\title{
Comparison of Static Incubation versus Physiologic Perfusion Techniques for Quantitation of Luminal Release of Prostacyclin and Thromboxane in Canine Arteries and Veins
}

\author{
Jan S. Brunkwall, M.D., ${ }^{1}$ James C. Stanley, M.D., Linda M. Graham, M.D., \\ William E. Burkel, Ph.D. AND David Bergqvist, M.D., Ph.D. \\ Section of Vascular Surgery, Department of Surgery, and the Department of Anatomy and Cell Biology, \\ University of Michigan Medical School, Ann Arbor, Michigan 48109-0331
}

Presented at the Annual Meeting of the Association for Academic Surgery, Orlando, FL, November 1-4, 1987

\begin{abstract}
Intraluminal release of 6-keto-PGF ${ }_{1 \alpha}$ and $\mathrm{TxB}_{2}$ in ex vivo canine arteries and veins was assessed during five consecutive 15-min periods using static incubation and physiologic perfusion techniques. Arterial segments were perfused with $90 \mathrm{ml} / \mathrm{min}$ pulsatile flow at $100 \mathrm{~mm} \mathrm{Hg}$ and vein segments with $90 \mathrm{ml} / \mathrm{min}$ nonpulsatile flow at $7 \mathrm{~mm} \mathrm{Hg}$. During the final 15 -min period vessels were stimulated with arachidonic acid (AAS). Perfusion of vein segments caused a higher release of 6-keto-PGF ${ }_{1 \alpha}$ during the first $30 \mathrm{~min}(P<0.05)$ and following AAS $(P<0.05)$ than did static incubation. Perfused arterial segments exhibited a higher relcasc than did incubated segments of 6-keto-PGF ${ }_{1 \alpha}$ for $45 \min (P<0.01)$ as well as following AAS $(P<0.01)$. TxB ${ }_{2}$ release was higher during the entire observation period in perfused arteries and veins compared to incubated vessels $(P<0.01$ and $<0.05$, respectively). There was no correlation between the amounts of 6 -keto- $\mathrm{PGF}_{1 \alpha}$ or $\mathrm{TxB}_{2}$ released when comparing values obtained by one technique to values obtained by the other $(P>0.1)$. These data suggest that flow related shear stress alters vascular prostanoid production, and that such should be accounted for when interpreting results of studies on prostacyclin and thromboxane release from intact vessels. (c) 1988 Academic Press, Inc.
\end{abstract}

\section{INTRODUCTION}

Vascular wall prostanoid production is complex. Many factors are known to stimulate prostanoid production from endothelial cells, including trauma [1], ischemia [2], immunologic injury with complement activation [3], irradiation [4-6], chemical factors [7-9], and platelet activation [10]. Prostacyclin, a potent vasodilator and inhibitor of platelet aggregation, is produced primarily by endothelium. It appears to have its dominant action locally, inasmuch as measured plasma levels of prostacyclin are too low to inhibit platelet aggregation or cause vasodilation [11, 12]. Thromboxane, a potent platelet aggregator and vasoconstrictor, is produced predominantly by platelets, with

\footnotetext{
${ }^{1}$ Visiting Research Scientist from the Department of Surgery, University of Lund, Malmö General Hospital, Malmö, Sweden.
}

lesser amounts derived from endothelial cells.

Earlier investigations on prostanoid generating capacity of the vessel wall often yielded inconsistent results. In part, this was a reflection of differing techniques used in assays, such as use of incubation of vessel homogenates [13-15], whole vessels [16], endothelial surfaces [17], cultured endothelial cells [9, 18], as well as use of incubation or flow devices [10, 19-21]. There are few comparative studies of the different methods to assess prostanoid release. Data obtained by one method cannot be compared with data obtained by a different method. For example, in one study incubation of the whole vessel in comparison to perfusion of the lumen resulted in a higher release of prostacyclin and thromboxane, as well as a higher prostacyclin:thromboxane ratio [20], with no consistent correlation in prostanoid release occurring between these two methods [22]. In 
contrast, perfusion of cultured endothelial cells causes a higher release of prostacyclin than does static incubation [23]. In this regard most perfusion studies have utilized low volume flow and low pressure systems which do not reflect in vivo flow conditions. The objective of this investigation was to compare in vitro release of prostacyclin and thromboxane from artery and vein segments subjected to physiologic pressure and flow versus that following static incubation.

\section{MATERIALS AND METHODS}

Arteries and veins were obtained from 16 mongrel dogs. All studies were carried out in accordance with the "Principles of Laboratory Animal Care" and "Guide for the Care of and Use of Laboratory Animals" (NIH Publication No. 80-23, revised 1978).

Vessel harvest. Animals were anesthetized with pentobarbital $(20 \mathrm{mg} / \mathrm{kg})$, intubated, and mechanically ventilated. The external jugular veins or common carotid arteries were exposed through a midline anterior neck incision, and carefully dissected from surrounding tissues, with ligation of all branches. Vessels were then excised, following which they were rinsed and flushed with calcium and magnesium free Hanks' balanced salt solution (HBSS-CMF) at $20^{\circ} \mathrm{C}$ (GIBCO Laboratories, Grand Island, NY; pH 7.4, 290 mOsm). Excised veins contracted to approximately half of their in situ length and arteries to two-thirds of their original length. Each vessel was divided into two pieces of equal length (approximately $3 \mathrm{~cm}$ for veins and $2.5 \mathrm{~cm}$ for arteries). These segments were briefly stored in HBSS-CMF until being simultaneously mounted and extended to their original in situ length in a perfusion system.

Vessel perfusion. Half the artery and vein segments were perfused with $25 \mathrm{ml}$ of Hanks' balanced salt solution (GIBCO Laboratories, pH $7.4,290 \mathrm{mOsm}$ ) at $37^{\circ} \mathrm{C}$ for five $15-\mathrm{min}$ periods. The perfusate was collected after each study period and stored at $-70^{\circ} \mathrm{C}$ for subsequent prostanoid radioimmunoassay
(RIA). Arachidonic acid (AA) was added to the final 15 -min perfusate, providing a concentration of $4 \mu \mathrm{g} / \mathrm{ml}$. In order to simulate normal in vivo perfusion patterns, vein segments were perfused at $7 \mathrm{~mm} \mathrm{Hg}$ pressure with nonpulsatile flow of $90 \mathrm{ml} / \mathrm{min}$, and arterial segments were perfused at $100 \mathrm{~mm} \mathrm{Hg}$ pressure with pulsatile flow of $90 \mathrm{ml} / \mathrm{min}$. The exact length and luminal circumference of the vessels being tested were measured and the luminal surface area exposed to the perfusate was calculated.

Vessel incubation. The remaining half of the artery and vein segments were mounted in the perfusion system as noted above, but they were not perfused. In order to create a pressure of approximately $7 \mathrm{~mm} \mathrm{Hg}$ inside the segments, the system was filled with $5 \mathrm{ml}$ of HBSS with an attached column filled to 10 $\mathrm{cm}$ height. At the end of each 15-min study period, the incubate, including the fluid column, was slowly propagated forward to allow all the HBSS containing 6-keto-PGF ${ }_{1 \alpha}$ and $\mathrm{TxB}_{2}$ to be collected. The final incubate had AA added, to provide a concentration of $4 \mu \mathrm{g} / \mathrm{ml}$. As was the case with the flow studies, the incubate medium was collected and stored at $-70^{\circ} \mathrm{C}$ for later prostanoid RIA. Luminal surface area exposed to the incubate was calculated as described above.

Vessel preparation for microscopy. Specimens were prepared for scanning electron microscopy following perfusion or incubation by pressure fixation in $2.5 \%$ glutaraldehyde in $0.1 \mathrm{M}$ cacodylate buffer (veins at 7 $\mathrm{mm} \mathrm{Hg}$, arteries at $100 \mathrm{~mm} \mathrm{Hg}$ ). Vessel segments were then dehydrated in ethanol, incubated for $10 \mathrm{~min}$ in hexamethydisilazane (HMDS), dried in air, and sputter coated with gold-palladium [24].

Prostacyclin and thromboxane assays. Prostanoids were extracted from the HBSS according to a modification of the method described by Simmons [25]. Briefly, acetonitrile was added to the perfusate solution to precipitate proteins which were then discarded. The supcrnatant was acidified with formic acid and mixed with ether to remove 
the lipids, from which the prostaglandins were extracted three times into ethyl acetate, dried in air, and then redissolved in $0.1 \%$ porcine skin-gel-phosphate-buffered saline. All studies were performed in duplicate.

Prostacyclin was measured as its stable degradation product 6 -keto- $\mathrm{PGF}_{1 \alpha}$ using a commercial radioimmunoassay kit (New England Nuclear, Boston, MA). This method employed an overnight incubation of ${ }^{3} \mathrm{H}$-labeled 6-keto-PGF ${ }_{1 \alpha}$ and a test sample with a constant amount of specific antibody raised against a BSA conjugate. The bound 6-keto-PGF ${ }_{1 \alpha}$ was absorbed with an activated charcoal suspension. The supernatant was then subjected to liquid scintillation with quantitation of $\beta$ activity. These values were compared with those from freshly made standard dilutions of 6-keto-PGF $1 \alpha$. The amounts of 6-keto-PGF $F_{1 \alpha}$ in the test sample were calculated by RIANAL computer analysis. The cross reactivity for other prostaglandins using this assay was: $\mathrm{PGF}_{2}=2.5 \%$, $\mathrm{PGF}_{1 \alpha}=0.3 \%, \mathrm{PGE}_{1}=0.2 \%, \mathrm{PGF}_{2 \alpha}$ $=0.05 \%$, and $\mathrm{TxB}_{2}<0.3 \%$.

Thromboxane was measured as its stable degradation product $\mathrm{TxB}_{2}$ according to a radioimmunoassay similar to that described above, also using ${ }^{3} \mathrm{H}$-labeled tracer as noted previously [26]. The cross-reactivity with other prostaglandins using this particular assay was $\mathrm{PGD}_{2}=1.0 \%, \mathrm{PGD}_{1}=0.32 \%$, $\mathrm{PGF}_{1 \alpha}=0.04 \%$, and $\mathrm{PGF}_{2 \alpha}=0.04 \%$ [26].

Prostanoid generation in this work is expressed as $\mathrm{pg} / \mathrm{mm}^{2}$ luminal area $/ 15 \mathrm{~min}$. Statistical significance of differences in prostanoid data was assessed using the Wilcoxon signed rank test.

\section{RESULTS}

Arteries. Incubation in arteries was associated with an initial burst release of 6-keto$\mathrm{PGF}_{1 \alpha}$ followed by a rapid decline after 30 min (Fig. 1) to approximately a third of the initial value $(P<0.01) .6-\mathrm{keto}^{-} \mathrm{PGF}_{1 \alpha}$ release then remained stable for $60 \mathrm{~min}$. With AA stimulation (AAS), there was a 2.2 -fold increase $(P<0.01)$. Perfusion, on the other

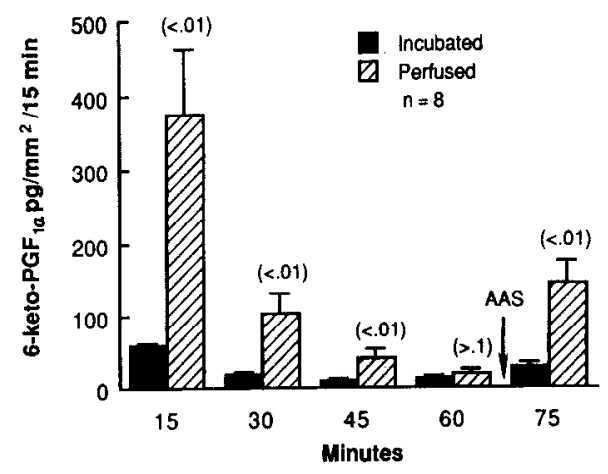

FIG. 1. Intraluminal release of 6-keto-PGF ${ }_{1 \alpha}$ in arteries: Effect of physiologic flow and pressure $(P=100 \mathrm{~mm}$ $\mathrm{Hg}, Q=90 \mathrm{ml} / \mathrm{min}$, pulsatile) and static incubation ( $P$ $=7 \mathrm{~mm} \mathrm{Hg}, Q=$ no flow).

hand, caused a 20-fold increase in 6-ketoPGF $_{1 \alpha}$ release during the first $15 \mathrm{~min}$ in contrast to later periods of perfusion $(P<0.01)$. Comparison of the two techniques revealed nearly an 8-fold higher initial release of 6keto-PGF ${ }_{1 \alpha}$ with perfusion $(P<0.01)$. Although this difference diminished with time, it remained significant at $45 \min (P<0.01)$. However, at $60 \mathrm{~min}$, release was the same regardless of which method was used $(P$ $=0.2$ ). With AAS in incubated arteries, release of 6-keto-PGF ${ }_{1 \alpha}$ increased approximately 2 -fold, whereas in perfused segments release increased approximately 8 -fold, a difference between the two techniques that was statistically significant $(P<0.01)$.

Release of $\mathrm{TxB}_{2}$ from incubated arteries (Fig. 2) paralleled the pattern of 6-keto$\mathrm{PGF}_{1 \alpha}$ release, although such occurred at much lower levels. This resulted in a relatively constant prostacyclin to thromboxane (P:T) ratio during the early observation periods. Release of $\mathrm{TxB}_{2}$ from perfused segments declined with time but was less than the decrease in 6-keto-PGF ${ }_{1 \alpha}$, which led to a decreased P:T ratio at 45 and $60 \mathrm{~min}$. AAS caused the P:T ratio to increase to its initial level $(P<0.01)$. $\mathrm{TxB}_{2}$ release was significantly greater from perfused segments than from incubated segments during the entire study $(P<0.01)$. This led to a slightly lower 


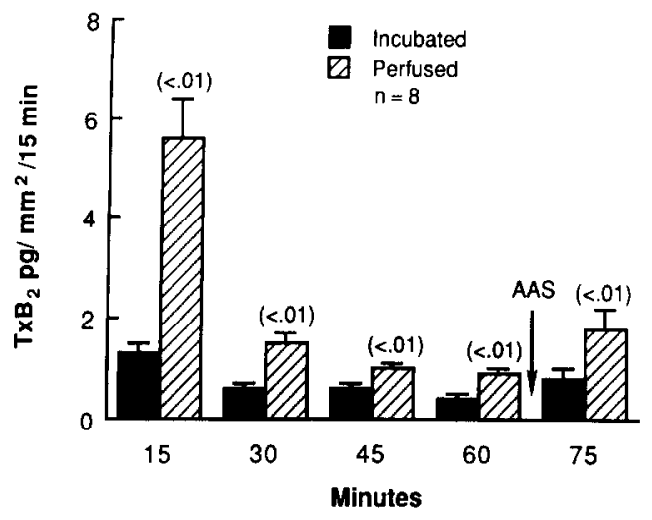

FIG. 2. Intraluminal release of $\mathrm{TxB}_{2}$ in arteries: Effect of physiologic flow and pressure $(P=100 \mathrm{~mm} \mathrm{Hg}, Q$ $=90 \mathrm{ml} / \mathrm{min}$, pulsatile $)$ and static incubation $(P=7 \mathrm{~mm}$ $\mathrm{Hg}, Q=$ no flow).

P:T ratio in perfused arteries when compared to incubated arteries, although such was statistically significant only after AAS $(P$ $<0.01$ ).

Veins. Static incubation of veins caused an immediate burst release of 6-keto-PGF ${ }_{1 \alpha}$ which decreased to two-thirds of the initial value after $15 \min (P<0.05)$, and remained relatively stable until $60 \mathrm{~min}$, at which time AAS caused a 2.6-fold increase (Fig. 3). Perfusion of veins also caused an initial burst release of 6-keto-PGF ${ }_{1 \alpha}$, with an 8-fold higher release during the first 15 min than at the 60 -min collection period $(P<0.005)$.

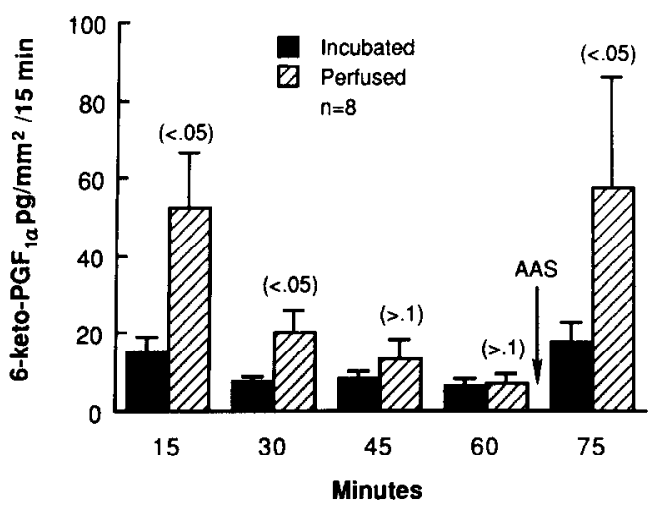

FIG. 3. Intraluminal release of 6-keto-PGF ${ }_{1 \alpha}$ in veins: Effect of physiologic flow and pressure $(P=7 \mathrm{~mm} \mathrm{Hg}, Q$ $=90 \mathrm{ml} / \mathrm{min}$, nonpulsatile $)$ and static incubation $(P=7$ $\mathrm{mm} \mathrm{Hg}, Q=$ no flow).
AAS of perfused veins produced an increase in 6-keto-PGF PG $_{1 \alpha}(P<0.005)$, to a level similar to that of the initial burst. Comparison of perfused versus incubated segments revealed a higher release from perfused vein segments for $30 \min (P<0.05)$ and following AAS $(P$ $<0.05$ ).

$\mathrm{TxB}_{2}$ release from incubated veins rapidly declined and remained stable after the first 15-min burst (Fig. 4). In contrast to 6-keto$\mathrm{PGF}_{1 \alpha}$, AAS failed to increase $\mathrm{TxB}_{2}$ release from incubated veins. Perfusion caused an initial burst release of $\mathrm{TxB}_{2}$ which decreased to about an eighth of the initial value by 60 $\min (P<0.005)$. AAS of perfused veins increased $\mathrm{TxB}_{2}$ release 2-fold $(P<0.01)$. $\mathrm{TxB}_{2}$ release was higher from perfused veins compared to incubated veins throughout the entire observation period $(P<0.05)$.

The P:T ratio from incubated veins was relatively stable during the study but did tend to be higher with AAS $(P<0.1)$. The P:T ratio from perfused veins was also stable for 60 min, but with AAS was significantly increased $(P<0.01)$. The P:T ratio was not statistically different between incubated and perfused vein segments $(P>0.1)$.

Attempts to relate quantities of 6-keto$\mathrm{PGF}_{1 \alpha}$ generated from incubation to quantities generated from perfusion did not reveal any correlation for release from either arter-

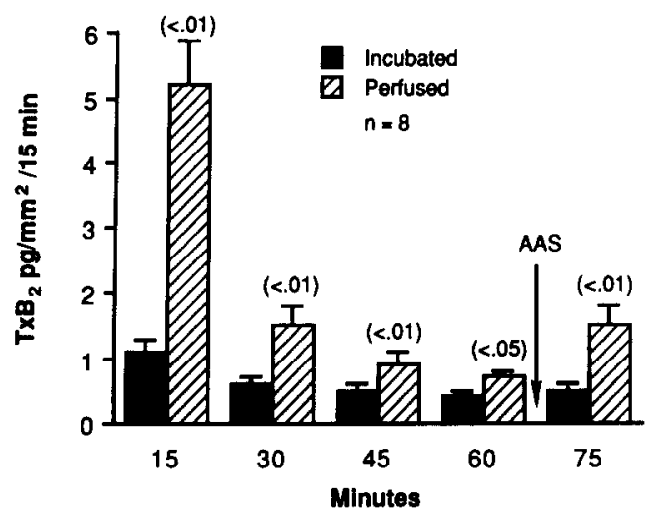

FIG. 4. Intraluminal release of $\mathrm{TxB}_{2}$ in veins: Effect of physiologic flow and pressure $(P=7 \mathrm{~mm} \mathrm{Hg}, Q=90$ $\mathrm{ml} / \mathrm{min}$, nonpulsatile) and static incubation $(P=7 \mathrm{~mm}$ $\mathrm{Hg}, Q=$ no flow). 
ies $(r=0.65, P=0.08)$ or veins $(r=0.44, P$ $=0.2$ ). Similarly, no correlation existed in $\mathrm{TxB}_{2}$ values when comparing data from incubation to perfusion methods in either arteries $(r<0.6, P>0.1)$ or veins $(r<0.5, P$ $>0.2$ ).

More than $90 \%$ of the luminal surfaces of both artery and vein specimens after $75 \mathrm{~min}$ of incubation or perfusion was covered with endothelial cells. No relation was found between differences in endothelial coverage and the amount of prostacyclin or thromboxane released. Solitary platelets were occasionally found along the surface, but there were no platelet aggregates observed in these vessels.

\section{DISCUSSION}

In determining the capacity of arteries and veins to release prostacyclin and thromboxane, it seems appropriate to use a technique that gives a maximal stimulation of the vessels without impairing their ability to produce prostanoids. Such a method should imitate events that occur in vivo. This current study clearly documents that veins and arteries subjected to physiologic pressure and flow conditions release higher amounts of prostacyclin and thromboxane than when exposed to conditions of static incubation. The higher initial release during the first 15 -min period might reflect release due to vessel manipulation during dissection, instrumentation, and cannulation. The fact that prostacyclin and thromboxane release was greater with perfusion than incubation, as well as with AAS supports the contention that this experimental model allows study of stimulated prostanoid release from arteries and veins.

Higher release of prostanoids from perfused vessels compared to incubated vessels may be explained in part by a number of factors. First, as proposed by Grabowski and colleagues [17], prostacyclin and thromboxane might be mixed more with the entire quantity of solution when perfused instead of staying in high concentrations at the interface of the endothelial cells with static incu- bation fluids. High concentrations at this interface may have an inhibitory effect on prostanoid release. A second factor relates to shear stress. Cultured endothelial cells exposed to increased shear stress are known to increase prostacyclin release [23, 27]. In the former studies the higher release was evident only during the first few minutes of increased shear stress, whereas in the present study a continuous increase was noted over the first $30 \mathrm{~min}$. This discrepancy may be related to differences in endothelial cells in culture compared to those in intact vessels, or to the pulsatile nature of flow with variable shear in the arterial segments. Shear stress appears to enhance prostanoid release, perhaps by mechanical stimulation, with increased phospholipid substrate or accelerated phospholipase or cyclooxygenase activity. In a previously published study from our laboratory, increases in pressure up to $100 \mathrm{~mm} \mathrm{Hg}$ enhanced initial release of prostacyclin in veins, but caused impaired responses to AAS [28]. In the current investigation the vein pressure was kept constant for both perfusion and incubation. Variables included a lesser amount of solution in direct contact with the vessel during incubation studies and the presence of flow in perfusion studies. In arterial studies, there was addition of pressure, flow, and pulsatility with perfusion.

The decline in prostanoid release over time in our studies may be speculated to be an effect of an accommodation of cells to new stimuli; a lack of phospholipids or an inhibition of phospholipid release leading to a lack of AA substrate; or an inhibition of cyclooxygenase activity. A loss of endothelium might also be implicated in that the presence of endothelial cells has been shown to be of importance for the response to externally provided AA $[10,29,30]$. However, over $90 \%$ of the vessel's endothelium remained intact in both perfused and incubated conduits. Therefore, the diminished prostanoid release with time is not due to loss of endothelial cells. Furthermore, the lack of platelets in both incubated and perfused segments excludes them as a source of $\mathrm{TxB}_{2}$. 
Prostacyclin release data from perfused arteries in the current experiment should not be rigidly compared to data from perfused veins. First, the segments were from different animals. Second, arterial segments in this particular study were from animals subjected to vein harvest for a different experiment, hours earlier. Third, they were perfused at different pressures. Fourth, arteries were perfused with a pulsatile flow whereas veins were perfused with a nonpulsatile flow. Fifth, the samples were run in separate RIA assays. However, the observed differences in prostacyclin release between arteries and veins in this study are consistent with those reported by others $[13,22]$. The overwhelming conclusion from reviewing existing data is that arteries respond with a higher release of prostacyclin compared to veins when exposed to physiological pressure and flow patterns, as well as when subjected to identical physical stresses.

Externally provided AA in the current experiment caused veins to release 6 -keto$\mathrm{PGF}_{1 \alpha}$ at the same level as during their initial perfusion. This is in contrast to arteries, which with AAS released about a third of their initial burst level. One explanation for this discrepancy might be that arteries possess more substrate than veins, but exhibit a more pronounced inhibition of cyclooxygenase or prostacyclin synthetase than do veins. A second explanation may be that arteries removed for study $3 \mathrm{hr}$ after an earlier vein harvest were stimulated in vivo, possibly by thrombin [8]. A third explanation relates to the possibility that the initial burst consists of prostacyclin from two different sources, of which only one responded to later AAS. Two such sources are endothelial cells and smooth muscle cells, the former responding to AAS with a 20 -fold greater release of prostacyclin $[10,29,31]$.

Greater release of both prostacyclin and thromboxane when perfused ex vivo compared to static incubation, only allows one to estimate the general responsiveness of vessels. Extrapolation of in vitro results to in vivo events must be undertaken cautiously, inasmuch as other factors probably affect prostaglandin synthesis, some of which undoubtedly have greater influence than those studied in this current experiment.

The more pronounced response to physiologic perfusion, together with the reproducible pattern of prostanoid release over time, causes us to favor the perfusion technique over the static incubation technique when assessing the luminal release of prostacyclin and thromboxane. The poor correlation between these two methods suggests that different regulatory mechanisms exist when vessels are perfused compared to when they are subjected to static incubation.

Certain conclusions should be apparent from this investigation. First, although statistically significant differences in prostanoid production of several magnitudes existed with incubation assays, the absolute amounts involved may not approach the quantities of prostaglandins released under physiologic conditions. As such, these differences are of limited relevance in understanding normal or abnormal vessel wall physiology. Second, because of the exceedingly disparate results of the methods studied, one must use great caution in interpreting previously reported prostanoid release data, especially those derived using incubation methodology.

\section{REFERENCES}

1. Brox, J. H., and Nordoy, A. Prostacyclin and ${ }^{51} \mathrm{Cr}$ release in cultured endothelial cells. Heamostasis 12: $345,1982$.

2. Needleman, P., Bronson, S. D., Wyche, A., Sivakoff, M., and Nicolaou, K. C. Cardiac and renal prostaglandin $\mathrm{I}_{2}$ : Biosynthesis and biological effects in isolated perfused rabbit tissues. J. Clin. Invest. 61: $839,1978$.

3. Goldsmith, J. C., and McCormick, J. J. Immunologic injury to vascular endothelial cells: Effects on release of prostacyclin. Blood 63: 984, 1984.

4. Eldor, A., Vlodavsky, I., Hy-Am, E., Atzmon, R., and Fuks, $Z$. The effect of radiation on prostacyclin $\left(\mathrm{PGI}_{2}\right)$ production by cultured endothelial cells. Prostaglandins 25: 263, 1983.

5. Hahn, G. L., Menconi, M. J., Cahill, M., and Polgar, $P$. The influence of gamma radiation on arachidonic acid release and prostacyclin synthesis. Prostaglandins 25: 783, 1983.

6. Sinzinger, H., Cromwell, M., and Firbas, W. Pros- 
tacyclin and thromboxan synthese der gëfasswand nach gammatron bestrahlung. Strahlentherapie Onkologie 162: 592, 1986.

7. Saba, S. R., and Mason, S. R. Studies of an activity from endothelial cells that inhibits platelet aggregation, serotonin release, and clot retraction. Thromb. Res. 5: 747, 1974.

8. Buchanan, M. R., Dejana, E., Cazenave, J. P., Mustard, J. F., and Hirsch, J. Uncontrolled $\mathrm{PGI}_{2}$ production by whole vessel wall segments due to thrombin generation in vivo and its prevention by heparin. Thromb. Res. 16: 551, 1979.

9. Weksler, B. B., Ley, C. W., and Jaffe, E. A. Stimulation of endothelial prostacyclin production by thrombin, trypsin, and the ionophore $\wedge 23187 . J$. Clin. Invest. 62: 923, 1978.

10. Papp, A. C., Crowe, L., Pettigrew, L. C., and Wu, K. K. Production of cicosanoids by dc-cndothclialized rabbit aorta: Interaction between platelets and vascular wall in the synthesis of prostacyclin. Thromb. Res. 42: 549, 1986.

11. Siess, W., and Dray, F. Very low levels of 6-ketoprostaglandin $\mathrm{F}_{1 a}$ in human plasma. J. Lab. Clin. Med. 99: 388, 1982.

12. Blair, I. A., Barrow, S. E., Wadell, K. A., Lewis, P. J., and Dollery, C. T. Prostacyclin is not a circulating hormone in man. Prostaglandins 23: 579, 1982.

13. Skidgel, R. A., and Priutz, M. P. PGI 2 production of rat blood vessels: Diminished prostacyclin formation in veins compared to arteries. Prostaglandins 16: 1,1978 .

14. Saroyan, R. M., Kerstein, M., Kadowitz, P. J., Hyman, A. L., and McNamara, D. B. Prostacyclin synthetase activity in diabetic human venous tissue. Surgery 96: 179, 1984.

15. Serneri, G. G. N., Abbate, R., Gensini, G. F., Panetta, A., Casolo, G. C., and Carini, M. TxA 2 production by human arteries and veins. Prostaglandins 25: 753, 1983.

16. Saldeen, P., Nilsson, I. M., and Saldeen, T. Increased synthesis of thromboxane $\mathrm{B}_{2}$ and 6-keto$\mathrm{PGF}_{1 \alpha}$ in hand veins from patients with deep venous thrombosis. Thromb. Res. 32: 461, 1983.

17. Grabowski, E. F., Naus, G. J., and Weksler, B. B. Prostacyclin production in vitro by rabbit aortic endothelium correction for unstirred diffusional layers. Blood 66: 1047, 1985.

18. Dejana, E., Prosdocimi, M., and deGaetano, G. Regulation of prostacyclin production by vascular cells in vitro and in vivo experimental systems. Wien. Klin. Woch. 98: 206, 1986.

19. Bjøro, K., Hovig, T., Stokke, K. T., and Stray-Pe- dersen, S. Formation of prostanoids in human umbilical vessels perfused in vitro. Prostaglandins 31: 683, 1986.

20. Mehta, P., Mehta, J., Crews, F., Roy, L., Ostrowski, N., and Horalek, C. Comparison of umbilical vein models for measurement of relative prostacyclin and thromboxane production. Prostaglandins 24: 743, 1982.

21. Mehta, P., Mehta, J., and Hay, D. Thromboxane and prostacyclin generation by intact human vessels in response to balloon catheter trauma. Prostaglandins Leukotrien. Med. 9: 539, 1982.

22. Brunkwall, J. S., Bergqvist, D., and Stjernquist, U. Prostacyclin and thromboxane release from the vessel wall-Comparison between an incubation and a perfusion model. Prostaglandins, in press.

23. Frangos, J. A., Eskin, S. G., and McIntire, L. V. Flow effects on prostacyclin production by cultured human endothelial cells. Science 227: 1477, 1985.

24. Nation, I. I.. A new method using hexamethyldisilazane for preparation of soft insect tissues for scanning electron microscopy. Stain Technol. 58: 347, 1983.

25. Simmons, P. M., Salmon, J. A., and Moncada, S. The release of leukotriene $B_{4}$ during experimental inflammation. Biochem. Pharmacol. 32: 1353, 1983.

26. Fitzpatrick, F. A., Gorman, R. R., McGuire, J. C., Kelly, R. C., Wynalda, M. A., and Sun, F. F. A radioimmunoassay for thromboxane $\mathrm{B}_{2}$. Anal. Biochem. 82: 1, 1977.

27. Grabowski, E. F., Jaffe, E. A., and Weksler, B. B. Prostacyclin production by cultured endothelial cell monolayers exposed to step increases in shear stress. J. Lab. Clin. Med. 105: 36, 1985.

28. Brunkwall, J. S., Stanley, J. C., Graham, L. M., and Burkel, W. E. Influence of pressure, flow rate, and pulsatility on release of 6-keto-PGFI $\mathrm{PI}_{1 \alpha}$ and $\mathrm{TxB}_{2}$ in ex vivo perfused canine veins. J. Vasc. Surg. 7: 99 , 1988.

29. Eldor, A., Falcone, D. J., Hajjar, D. P., Minick, C. R., and Weksler, B. Recovery of prostacyclin production by de-endothelialized rabbit aorta. J. Clin. Invest. 67: 735, 1981

30. Goldsmith, J. C. Contribution of the subendothelium to prostacyclin release after vascular injury. $J$. Lab. Clin. Med. 100: 574, 1982.

31. Boeynaems, J. M., Galand, N., and Ketelbant, P. Prostacyclin production by the de-endothelialized rabbit aorta. J. Clin. Invest. 76: 7, 1985. 